\title{
Migration reversal of soft particles in vertical flows
}

\author{
Andre Förtsch, Matthias Laumann, Diego Kienle and Walter Zimmermann \\ Theoretische Physik I, Universität Bayreuth, 95440 Bayreuth, Germany
}

PACS 47.15.G - Low-Reynolds-number (creeping flows)

PACS 47.57.ef - Sedimentation and migration

PACS 83.50.-v - Deformation and flow

\begin{abstract}
Non-neutrally buoyant soft particles in vertical microflows are investigated. We find, soft particles lighter than the liquid migrate to off-center streamlines in a downward Poiseuille flow (buoyancy-force antiparallel to flow). In contrast, heavy soft particles migrate to the center of the downward (and vanishing) Poiseuille flow. A reversal of the flow direction causes in both cases a reversal of the migration direction, i. e. heavier (lighter) particles migrate away from (to) the center of a parabolic flow profile. Non-neutrally buoyant particles migrate also in a linear shear flow across the parallel streamlines: heavy (light) particles migrate along (antiparallel to) the local shear gradient. This surprising, flow-dependent migration is characterized by simulations and analytical calculations for small particle deformations, confirming our plausible explanation of the effect. This density dependent migration reversal may be useful for separating particles.
\end{abstract}

Introduction. - Microfluidics is a rapidly evolving cross-disciplinary field, ranging from basic physics to a great variety of applications in life science and technology [1 9]. The blooming subfield of the dynamics of neutrally buoyant soft particles in suspension and their crossstreamline migration (CSM) in rectilinear shear flows, plays a central role for cell and DNA sorting, blood flow, polymer processing and so on $6,10-13$. In contrast, little is known about the dynamics of non-neutrally buoyant soft particles in rectilinear flows, but we show in this work for such particles a novel migration reversal.

Segre and Silberberg reported in 1961 about CSM of neutrally buoyant rigid particles at finite Reynolds numbers in flows through pipes [14]. When particles and channels approach the micrometer scale, fluid inertia does not matter and particles follow the Stokesian dynamics. In this limit CSM occurs only for soft particles but in curvilinear 15 17] as well as in rectilinear flows 18 20], whereby in rectilinear flows, the flows fore-aft symmetry is broken, requiring intra-particle hydrodynamic interaction 18, 19]. Such symmetry breaking occurs also near boundaries via wall-induced lift forces $20-24$ or by space-dependent shear rates, so that dumbbells [18, 19], droplets 25, 26], vesicles and capsules [27, 29] exhibit CSM even in unbounded flow. Such parity breaking mechanisms may be also accompanied by a viscosity contrast 30 or chirality 31. Recently was found, that CSM takes place also for asymmetric soft particles in time-dependent linear shear flow 32 and that soft particles are actuated even in a homogeneous but time-dependent flow by taking particle inertia into account 33 .

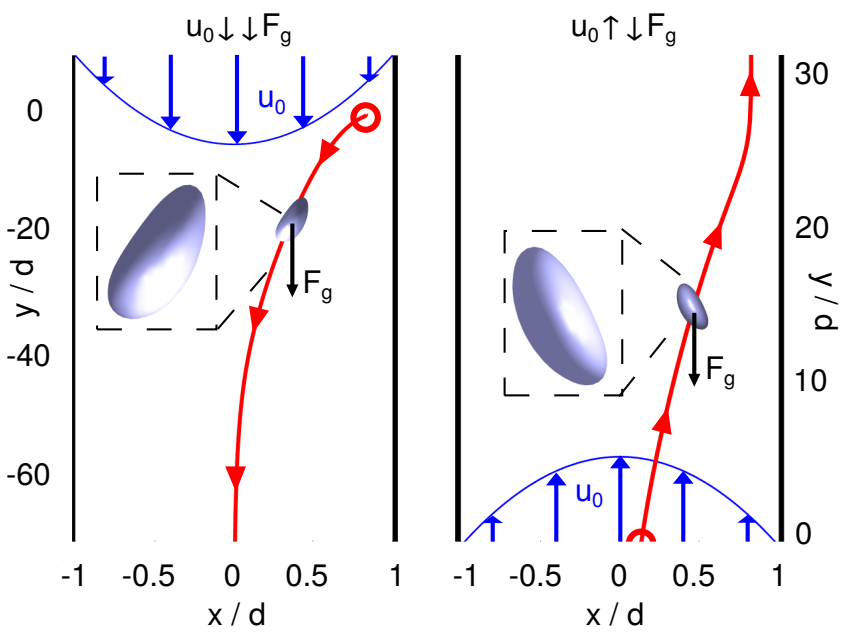

Fig. 1: The left part shows in red the center of mass trajectory, $\left(x_{c}(t), y_{c}(t)\right)$, of a heavy and soft tank-treadin capsule in a downward Poiseuille flow, $\boldsymbol{u}_{0}(x)$, parallel to the gravitational force $\boldsymbol{F}_{g}: \boldsymbol{u}_{0} \downarrow \downarrow \boldsymbol{F}_{g}$. The right part shows a trajectory (red) of a heavy capsule in an upward Poiseuille flow, i. e. $\boldsymbol{u}_{0} \uparrow \downarrow$ $\boldsymbol{F}_{g}$, where the capsule migrates away from the center of the Poiseuille flow. The two confining walls are at a distance $2 d$.

Heavy rigid particles in a finite Reynolds number flow 
downward in a gravitational field migrate away from the tube center and for an upward flow to the tube center, as experimentally observed 34 . Effects of axial forces on rigid particles along the tube axis in finite Reynolds number flows where also studied in Refs. 35.36 and effects of axial (electrical) forces on (charged) polymers in in pipe flows in Refs. 37, 38. Little is known about CSM of nonneutrally buoyant soft particles in vertical Stokes flows.

Here we show that a soft heavy particle migrates to the center of a tube in the limit of a vanishing Reynolds number, while rigid particles don't 39. Furthermore is shown, that heavy (light) soft particles migrate in vertical rectilinear Stokes flows antiparallel (parallel) to the shear gradient. This dependence of the CSM direction on the shear gradient is shown by approximate analytical calculations and by numerical simulations for soft capsules and ring polymers. Also a plausible qualitative explanation of the origin migration of non-neutral particles is provided: It is based on the interplay between the orientation of the shear induced elliptical shaped soft capsule (ring) together with the related anisotropic friction, a non-buoyant particle experiences.

Modeling soft particles in Stokes flow. - The dynamical equations of two non-neutrally buoyant particles, bead-spring models for ring polymers and elastic capsules in rectilinear flows is described in this section. The unperturbed linear shear flow is given by $\boldsymbol{u}_{0}(x)=\dot{\gamma} x \mathbf{e}_{y}$ and the Poiseuille flow between the two confining plane boundaries at $x_{d}= \pm d$ by

$$
\boldsymbol{u}_{0}(x)=\hat{u}_{0}\left(1-\frac{x^{2}}{d^{2}}\right) \mathbf{e}_{y} .
$$

The maximal velocity $\hat{u}_{0}$ at the center $y=0$ and the shear rate $\dot{\gamma}$ can be either positive or negative.

The migration of the soft particles is obtained by their non-Brownian trajectories. The trajectories of the beadspring ring-polymer and the capsule in an unperturbed $\boldsymbol{u}_{0}(x)$ are determined by solving the standard Stokesian dynamics for bead-spring models with the position $\boldsymbol{r}_{i}$ of the $i$-th bead:

$$
\dot{\boldsymbol{r}}_{i}=\boldsymbol{u}_{0}\left(\mathbf{r}_{i}\right)+\sum_{j=1}^{N} \mathbf{H}_{i j} \boldsymbol{F}_{j}
$$

$\boldsymbol{F}_{j}$ describes the force acting on the $j$-th bead and $\mathbf{H}_{i j}$ is the mobility matrix described in the following.

The harmonic spring potential for a ring polymer with a finite mean distance $b$ between next-neighbor beads and spring constant $k$ is given by:

$$
V_{s p r}=\frac{k}{2} \sum_{i=1}^{N}\left(b-r_{i j}\right)^{2} .
$$

Due to the bending potential

$$
V_{b}=-\frac{\kappa}{2} \sum_{i=1}^{N} \ln \left(1+\cos \beta_{i}\right)
$$

with the bending constant $\kappa$ the closed polymer has in the undeformed state the shape of a ring. The angle $\beta_{i}$ is given by $\cos \left(\beta_{i}\right)=\hat{\boldsymbol{r}}_{(i-1) i} \cdot \hat{\boldsymbol{r}}_{i(i+1)}$ and the distance vector between the beads by $\boldsymbol{r}_{i, j}=\boldsymbol{r}_{i}-\boldsymbol{r}_{j}=r_{i, j} \hat{\boldsymbol{r}}_{i, j}$. Unit vectors are denoted by a hat. The forces $\boldsymbol{F}_{j}$ in Eq. (2) are given by $\boldsymbol{F}_{j}=-\nabla_{j}\left[V_{s p r}+V_{b}\right]+\boldsymbol{F}_{g}$, with $\boldsymbol{F}_{g}=F_{g} \mathbf{e}_{y}$.

$H_{i j}$ is the mobility matrix describing the hydrodynamic interactions between beads in the presence of a single wall parallel to $y z$-plane with no-slip boundary condition, which is of the following form [40]:

$$
\begin{aligned}
H_{i j}\left(\boldsymbol{r}_{i}, \boldsymbol{r}_{j}\right) & ={ }^{S} H_{i j}-{ }^{S} H_{i j}\left(\boldsymbol{r}_{i}, \boldsymbol{r}_{j}^{\prime}\right) \\
& +{ }^{D} H_{i j}\left(\boldsymbol{r}_{i}, \boldsymbol{r}_{j}^{\prime}\right)-{ }^{S D} H_{i j}\left(\boldsymbol{r}_{i}, \boldsymbol{r}_{j}^{\prime}\right) .
\end{aligned}
$$

Herein $\boldsymbol{r}_{j}^{\prime}=\left(x_{j}+2 h_{j}, y_{j}, z_{j}\right)$ is the position of a mirrorparticle to the $j$-th bead with distance $h_{j}$ to the wall. The first term contains the hydrodynamic interaction (HI) in the bulk regime, represented by the Oseen tensor 41]

$$
{ }^{S} H_{i j}^{\alpha \beta}\left(\boldsymbol{r}_{i}, \boldsymbol{r}_{j}\right)=\left\{\begin{array}{c}
\frac{1}{8 \pi \eta r_{i j}}\left(\delta_{\alpha \beta}+\frac{r_{i j}^{\alpha} r_{i j}^{\beta}}{r_{i j}^{2}}\right) \quad i \neq j \\
\frac{1}{6 \pi \eta a} \delta_{\alpha \beta}
\end{array}\right.
$$

where $\alpha, \beta \in\{x, y, z\}$ and $6 \pi \eta a$ is the Stokes friction with the bulk viscosity $\eta$ and bead radius $a$. The second term in Eq. (5) is the HI generated by the mirror image

$$
{ }^{S} H_{i j}^{\alpha \beta}\left(\boldsymbol{r}_{i}, \boldsymbol{r}_{j}^{\prime}\right)=\frac{1}{8 \pi \eta \tilde{r}_{i j}}\left(\delta_{\alpha \beta}+\frac{\tilde{r}_{i j}^{\alpha} \tilde{r}_{i j}^{\beta}}{\tilde{r}_{i j}^{2}}\right),
$$

with $\tilde{\boldsymbol{r}}_{i j}=\boldsymbol{r}_{i}-\boldsymbol{r}_{j}^{\prime}=\tilde{r}_{i j} \hat{\tilde{\boldsymbol{r}}}_{i j}$ is the distance to the mirror bead image $j$. The last two terms in (5) contain the Stokes doublet (D) part

$$
{ }^{D} H_{i j}^{\alpha \beta}\left(\boldsymbol{r}_{i}, \boldsymbol{r}_{j}^{\prime}\right)=\frac{h_{j}^{2}\left(1-2 \delta_{\beta y}\right)}{4 \pi \eta \tilde{r}_{i j}^{3}}\left(\delta_{\alpha \beta}-3 \frac{\tilde{r}_{i j}^{\alpha} \tilde{r}_{i j}^{\beta}}{\tilde{r}_{i j}^{2}}\right),
$$

and the source doublet (SD) part

$$
\begin{aligned}
& { }^{S D} H_{i j}^{\alpha \beta}\left(\boldsymbol{r}_{i}, \boldsymbol{r}_{j}^{\prime}\right)=\frac{1}{4 \pi \eta \tilde{r}_{i j}^{3}} h_{j}\left(1-2 \delta_{\beta y}\right) \\
& \left(\delta_{\alpha \beta} \tilde{r}_{i j}^{y}-\delta_{\alpha y} \tilde{r}_{i j}^{\beta}+\delta_{\beta y} \tilde{r}_{i j}^{\alpha}-3 \frac{\tilde{r}_{i j}^{\alpha} \tilde{r}_{i j}^{\beta} \tilde{r}_{i j}^{y}}{\tilde{r}_{i j}^{2}}\right)
\end{aligned}
$$

of the HI. To take account of the effects of the second wall, a superposition of two single walls is used. This approximation generates according to [42] reasonable results, if the particle size to channel-width ratio is less than 5 . In simulations without walls only ${ }^{S} \boldsymbol{H}_{i j}$ is used.

In case of the capsule we use the same equations of motion, but different potential forces. The elastic forces of the capsule are described by the Neo-Hookean Law with Potential $V_{N H}$. The Neo-Hookean Law describes a rubber like material with a constant surface shear elastic modulus $G$ 43, 44. 
Furthermore we use a bending-potential 45

$$
V_{h}=-\frac{\kappa}{2} \sum_{i, j}\left(1-\cos \beta_{i, j}\right),
$$

where $\beta_{i, j}$ is the angle between two normal vectors of neighboring triangles of beads, and a potential to conserve the volume of the capsule 45 .

$$
V_{V}=-\frac{k_{v}}{V_{0}}\left(V(t)-V_{0}\right)^{2} .
$$

$V(t)$ means the Volume at a given time $\mathrm{t}$ and $V_{0}=\frac{4}{3} \pi R^{3}$ the desired Volume of the capsule (with the Radius $\mathrm{R}$ of the not deformed spherical capsule). The force in case of the capsule is given by $\boldsymbol{F}_{i}=-\nabla_{i}\left[V_{h}+V_{V}+V_{N H}\right]$

If not stated otherwise we use the following parameters. For the flow, $u_{0}=0.5, d=60, \eta=1.0$; the ring, $a=0.5$, $k=0.175, \kappa=6.0, N=16, b=2.5$ (which gives a ring radius of $R=6.36$ ); the capsule: $G=0.1, \kappa=0.1$, $k_{v}=3.0, a=0.4, N=642, b=1.0$ (which gives a capsule's radius $R=6.6$ ); vertical force $\boldsymbol{F}_{g}=-0.01 \hat{\mathbf{e}}_{y}$.

Qualitative explanation of cross-stream migration in vertical flows. - A capsule or a ring-polymer model exposed to a linear shear or a Poiseuille flow is deformed by the local shear gradient, as shown in Fig. 2. but the capsule's (ring's) shape is not identical in a linear shear and a Poiseuille flow (here demonstrated for moderate local shear rates). However, for flows the capsule (ring) may be described in a first approximation by a rotational symmetric ellipsoid (elliptical polymer) with a Stokes drag-coefficient $\zeta_{\perp}\left(\zeta_{\|}\right)$in the direction perpendicular (parallel) to the major axis and $\zeta_{\perp}>\zeta_{\|}[44$.

The major axis of a tank-treading capsule (ring) includes with the straight flow lines of both flows an angle, whereby the sign and the magnitude of this angle are determined by the sign and the magnitude of the local shear rate 44]. The buoyancy force acting on a particle points upward for a light and downward for a heavy particle, i. e. it is either parallel or antiparallel to the flow lines. According to different drag coefficients, $\zeta_{\perp}>\zeta_{\|}$, an external force on an inclined capsule (ring) in shear flow causes an oblique migration velocity $\mathbf{v}_{m}$, as shown in Fig. 2. The inclination angle of the ellipsoid (ring) and therefore the inclination angle $\alpha$ of $\mathbf{v}_{m}$ depend on the sign of the local shear rate (see also analytical results below). For both flows a reversal of the flow direction leads to a reversal of the local shear gradient and simultaneously to a reversal of the horizontal component ( $x$ component) of the migration velocity $\mathbf{v}_{m}$ : I. e. a reversal of the flow direction causes a reversal of the cross-streamline migration of non-neutrally buoyant soft particles. Furthermore, if the buoyancy force is downward (upward) then the shear gradient and the horizontal migration direction are antiparallel (parallel).

Small capsule-deformations. - The shape of a Neo-Hookean capsule, its anisotropic drag and its crossstreamline drift in a linear shear flow $\boldsymbol{u}_{0}=\dot{\gamma} x \hat{e}_{y}$ can be
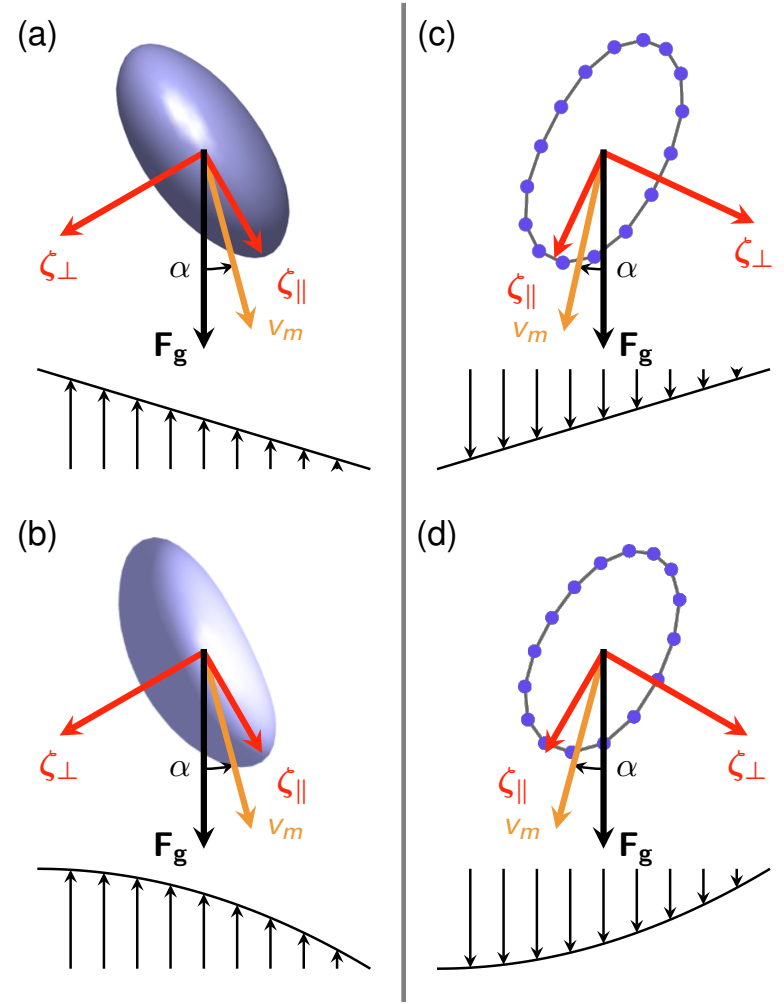

Fig. 2: Soft capsules and rings are deformed by shear flows as indicated in (a)-(d) for moderate local shear rates. The particle's deformation in a linear shear and a Poiseuille flow is slightly different as indicated by the differences between the shapes in (a) and (b) for the capsule in (c) and (d) for the ring. The particle's major axis in shear flow is inclined with respect to the flow lines and the particles has different Stokes drag coefficients $\zeta_{\|}$parallel and $\zeta_{\perp}$ perpendicular to the major axis. Therefore, if an external force $\boldsymbol{F}_{g}$ acts on the particle (parallel or antiparallel to the flow direction) the resulting particle migration velocity $v_{m}$ encloses an angle $\alpha$ with the straight flow lines and leads to cross-stream line migration (CSM). If $\boldsymbol{F}_{g}$ points downward (upward) then the horizontal (cross-streamline) component of $\mathbf{v}_{m}$ and the shear gradient have opposite (equal) sign. Accordingly, for a given force $\boldsymbol{F}_{g}$ a reversal of the flow direction leads to a reversal of the shear gradient and therefore of the particle's (horizontal) CSM direction.

determined analytically in the range of a small capillary number $\mathrm{Ca}=\dot{\gamma} \frac{\eta R}{G} 43,44$. In this limit, the capsule shape is given by the equation

$$
\begin{aligned}
r^{2} & =x^{2}+y^{2}+z^{2}=R^{2}+\frac{5}{3} \frac{\mathrm{Ca}}{\dot{\gamma}} \boldsymbol{r}^{T} \cdot \boldsymbol{J} \cdot \boldsymbol{r}+O\left(\mathrm{Ca}^{2}\right) \\
\boldsymbol{J} & =\frac{1}{2}\left[\left(\nabla \otimes \boldsymbol{u}_{0}\right)+\left(\nabla \otimes \boldsymbol{u}_{0}\right)^{T}\right] \\
\boldsymbol{r} & =(x, y, z)^{T}
\end{aligned}
$$

which describes an ellipsoid with three different axes. The major axis forms with the undisturbed straight stream lines an angle of about $\frac{\pi}{4}$. The length of the three axes 
are

$$
\begin{aligned}
d_{1,3} & \left.=\frac{\sqrt{2}}{\sqrt{2 \mp \frac{25}{3} \mathrm{Ca}}} R, \quad \text { (major } / \text { minor axis }\right) \\
d_{2} & =R .
\end{aligned}
$$

In order to proceed with analytical calculations we make a common approximation and assume rotational symmetry with respect to the major axis. Then the length of the major/minor axis are given by

$$
\begin{array}{ll}
a=\frac{d_{2}+d_{3}}{2}, & \\
b=d_{1} . & \text { (two minor axes) }
\end{array}
$$

The drag coefficients of a rotational symmetric ellipsoid parallel and perpendicular to the major axis are given by Perrin's formulas (see e.g. [46] and references therein)

$$
\begin{aligned}
\zeta_{\perp} & =\frac{8}{3} \frac{1}{\frac{\beta}{\beta^{2}-1}+\frac{\left(2 \beta^{2}-3\right) \ln \left(\beta+\sqrt{\beta^{2}-1}\right)}{\left(\beta^{2}-1\right)^{\frac{3}{2}}}}, \\
\zeta_{\|} & =\frac{8}{3} \frac{1}{\frac{2 \beta}{1-\beta^{2}}+\frac{\left(2 \beta^{2}-1\right) \ln \left(\frac{\beta+\sqrt{\beta^{2}-1}}{\beta-\sqrt{\beta^{2}-1}}\right)}{\left(\beta^{2}-1\right)^{\frac{3}{2}}}}
\end{aligned}
$$

with $\beta=\frac{b}{a}$. The migration velocity of the ellipsoid perpendicular and parallel to the stream lines is obtained by decomposing the buoyancy fore $\boldsymbol{F}_{g}=F_{g} \hat{\mathbf{e}}_{y}$ into its component along the major axis, $\mathbf{F}_{g, \|}$, and perpendicular to it, $\mathbf{F}_{g, \perp}$. The migration velocity across the streamlines is then given by

$$
v_{m}=\left(\frac{\boldsymbol{F}_{g, \perp}}{\zeta_{\perp}}+\frac{\boldsymbol{F}_{g, \|}}{\zeta_{\|}}\right) \cdot \hat{\mathbf{e}}_{x} .
$$

A Taylor expansion with respect to $\mathrm{Ca}$ gives at leading order

$$
v_{m}=\frac{5}{96} \frac{F_{g}}{\pi \eta R} \mathrm{Ca}+O\left(\mathrm{Ca}^{2}\right) .
$$

The capsule's shape in a Poiseuille flow at an off-center position has the shape of a slightly deformed ellipsoid, as indicated also in Fig. 2, The deformation of a spherical shape is determined by the local shear rate in Poiseuille flow at the capsule's center $\left(x_{c}, y_{c}\right): \dot{\gamma}=-\frac{2 u_{0} x_{c}}{d^{2}}$. With the local capillary number $\mathrm{Ca}=\dot{\gamma} \frac{\eta R}{G}=-\frac{2 u_{0} x_{c} \eta R}{d^{2} G}$ one obtains within this approximation the position dependent cross-streamline migration velocity in Poiseuille flow

$$
v_{m} \approx-\frac{5}{48} \frac{F_{g} u_{0} x_{c}}{\pi G d^{2}}
$$

The force induced velocity $v_{y}=\left(\mathbf{v}_{m}\right)_{y}$ relative to the unperturbed flow can be calculated analogous as in a linear shear, which includes besides result from the Stokes drag a deformation dependent correction proportional to $\mathrm{Ca}$ :

$$
v_{y}=\frac{F_{g}}{6 \pi \eta R}+\frac{5}{288} \frac{F_{g}}{\pi \eta R} \mathrm{Ca}+O\left(\mathrm{Ca}^{2}\right) .
$$

Its explicit form for Poiseuille flow is then

$$
v_{y} \approx \frac{F_{g}}{6 \pi \eta R}-\frac{5}{144} \frac{F_{g} u_{0} x_{c}}{\pi d^{2} G}
$$

If the external force is antiparallel to the flow and if it is large enough, the capsule moves against the flow direction. This is approximately the case if $v_{y}$ is larger then the velocity of the Poiseuille flow at the capsule center

$$
\begin{aligned}
v_{y} & >u_{0}\left(x_{x}, y_{c}\right) \\
\frac{F_{g}}{6 \pi \eta R}-\frac{5}{144} \frac{F_{g} u_{0} x_{c}}{\pi d^{2} G} & >u_{0}\left(1-\frac{x^{2}}{d^{2}}\right) \\
F_{g} & >\frac{144 \pi \eta R d^{2} G u_{0}}{24 d^{2} G-5 R \eta u_{0} x_{c}}\left(1-\frac{x_{c}^{2}}{d^{2}}\right)
\end{aligned}
$$

Numerical results on CSM in unbounded flows. - To verify our approximate analytical predictions and qualitative descriptions of the migration of non-neutrally buoyant soft particles in unbounded flows, we simulate the Stokesian dynamics of capsules and a rings.

This ensures that the occurred migration is no inertial effect or imposed by wall interactions. Since the migration reversal does only depend on the local shear rate, we confine the numerical investigation, without loss of generality, to that of the Poiseuille flow.

The setup for all simulations can be seen in Fig. 1. The imposed flow is a Poiseuille flow applied in y-direction with $-d<x<d$. The deformable particles are placed with center of mass positions $x_{c}$. As consequence, particles with negative migration velocity $\left(v_{m}<0\right)$ will migration to the wall and positive migration means center migration.

As reference, we first discuss the case without external force, which is shown as red curve in Fig. 3. For this case we observe the already known center migration for both, the capsule and the ring [zitat: zentrumsmigration fr soft-particle low Re].

If we now apply a gravitational force parallel to the stream lines $\left(\boldsymbol{u}_{0} \downarrow \boldsymbol{F}_{g}\right)$, we see an increase of the center migration (Fig. 3 (a) and (c)). This additional centermigration is enlarged for higher external forces. During the migration to the center, the migration speed decreases, which is a result of the position dependent shear-rate of the Poiseuille flow. When the particles reach the center line, $v_{m}$ vanishes and the particle follows the stream lines.

For the case of antiparallel force and flow-direction $\left(\boldsymbol{u}_{0} \uparrow \downarrow \boldsymbol{F}_{g}\right)$, we find the opposite behavior (Fig. 3 (b) and (d)). The migration direction is reversed and the particles experience a positive migration-velocity. This effect is also correlated to the force strength and position of the particle. The migration in this case does not vanish on its way remote from the center, since there is no repulsive interaction with the walls. This migration reversal is observed for both, the capsule and the ring. Both migration curves look similar, which means the reversal effect does not depend on the detail of the particle but is a more 
generic feature of the interplay between deformation and external forces. This is consistent with the results from 37, where they could measure this behavior for bundled DNA-molecules, which have no impenetrable surface.

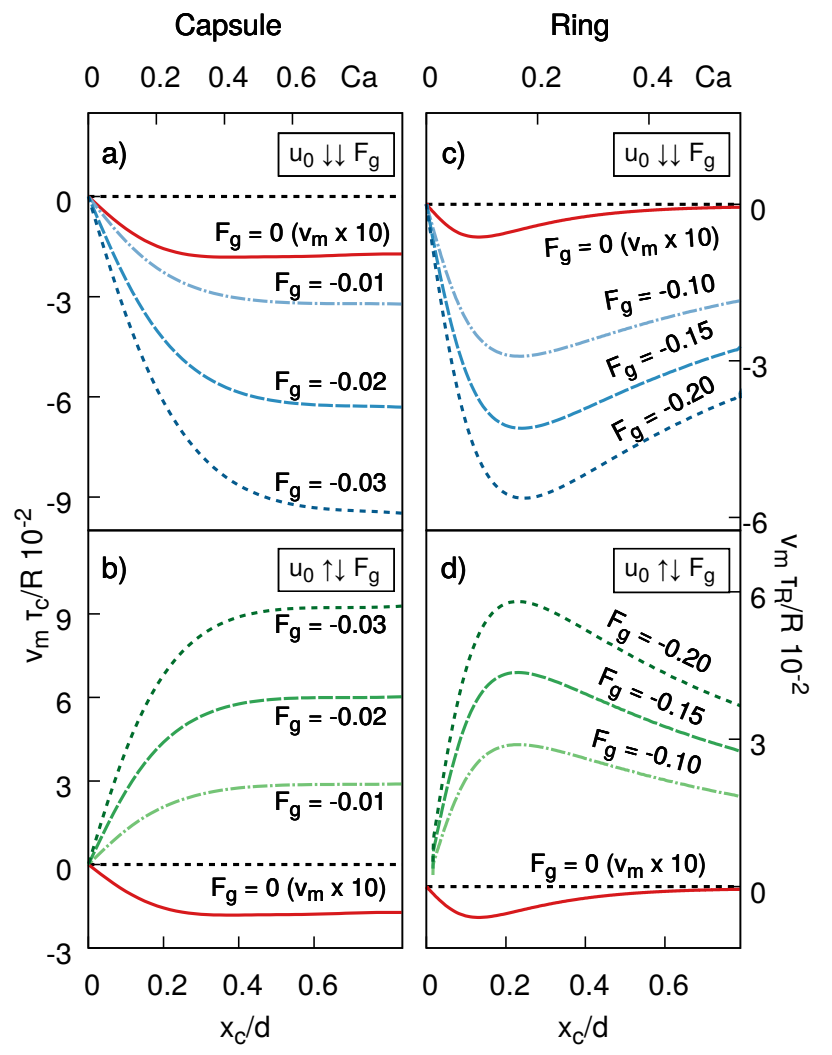

Fig. 3: Cross-streamline migration-velocity $v_{m}$ of deformable capsules [a) and b)] and rings [c) and d)] in unbounded vertical Poiseuille flows as function of the capillary number Ca and for different values of the vertical force $F_{g}$. For parallel flow and force directions, $\mathbf{u}_{0} \downarrow \downarrow \mathbf{F}_{g}$, the particles migrate to the center of the Poiseuille flow, i. e. $v_{m}<0\left(v_{m}>0\right)$ in range $x_{c}>0$ $\left(x_{c}<0\right)$, only faster than in the case of neutral particles with $\mathbf{F}_{g}$. For an antiparallel configuration, $\mathbf{u}_{0} \uparrow \downarrow \mathbf{F}_{g}$, the migration is reversed and away from the center of Poiseuille flow. These results show, that force induced relative velocity $\mathbf{v}_{m}$ in Fig. 2 increases with the magnitude of $F_{g}$.

\section{Cross-stream drift between walls. -}

Migration of a sedimenting capsule. The influence of the flat walls of the channel should also be studied. At first we investigate the interaction between a sedimenting capsule and the walls of the channel without a flow. We observe a repulsion of the capsule from the wall which depends on the stiffness of the capsule, see Fig. 4. The softer the capsule is the stronger is the repulsion. This is consistent with the fact that a solid particle sinks parallel to the wall (see zitat).

The reason of the repulsion is the deformability of the capsule. The part of the capsule closer to the wall is subjected to a friction with the wall (transmitted via the fluid) and lags behind. The other part of the capsule more away from the wall moves therefore faster. This stretches the capsule and lasts until a steady state is reached which has a shorter and a longer axis. The longer axis points away from the wall if seen from the center of the capsule in direction of the external force. This leads, as described above, to an asymmetric drag and the capsule moves not completely in direction of the force but a bit shifted towards the major axis. This means it drifts away from the wall. This mechanism is different from the wall repulsion of capsules in a shear flow without a external force where a lift force due to the tank treading leads to the wall repulsion.

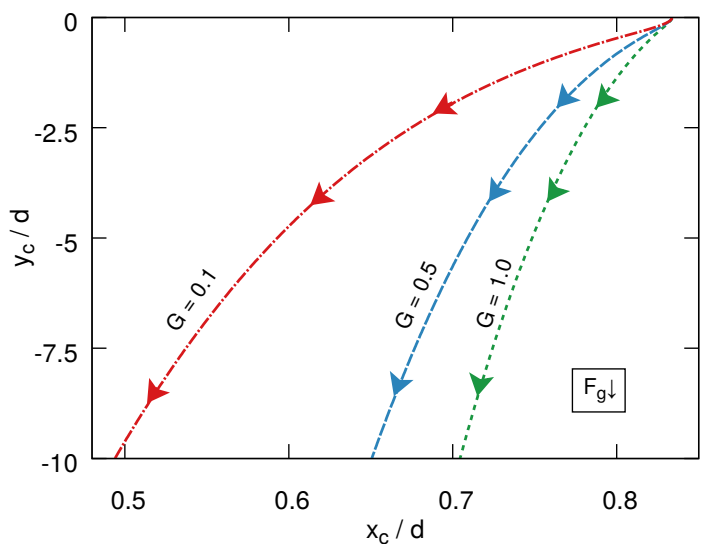

Fig. 4: Trajectories of a deformable capsule sedimenting between two walls in the absence of flow $\left(\boldsymbol{u}_{0}=0\right)$ for three different values of the Neo-Hookean stiffness $G$. Softer particles move faster away from the wall (at $\left.\frac{x}{d}=1.0\right)$ and rigid particles don't.

Dynamics of rings and capsules in Poiseuille flows. The contribution of the wall interaction to the migration of capsules in a Poiseuille flow is examined here. The Fig. 3 and 5 show the migration velocity $v_{m}$ of a ring and a capsule as function of the capillary number Ca for different values of the force $F_{g}$ in case of an unbounded or an bounded flow. A comparison of both Fig. shows that far away from the walls the migration is similar in case with and without walls but is changed close to the walls where the repelling lift force becomes important. In case of a parallel external force and flow the lift force enhances the migration to the center. In case of an antiparallel external force and flow the bulk migration to the wall is hindered or surpassed by the lift force. The capsule and the ring migrates away from the wall if it is close to it. This leads to stable lateral position $x_{e q}$ outside the channel center where the migration due to the external force and due to the the lift force are equal.

The drift towards such a stable wall distance at $x_{e q} \approx$ $0.85 d$ and the dependence of $x_{e q}$ on $u_{0}$ and $F_{g}$ is shown in Fig. 6. If the flow is parallel to the force $u_{0}<0$ the stable position is the channel center because the external force, the center migration occurring also without an external force and the wall repulsion lead to a migration to the center. If the flow is reversed (meaning now antipar- 
allel to the force) the migration due to the force leads to a wall migration and stable, lateral off-center positions occur. They are at approximately $u_{0}=0.05$ the closest to the wall depending on the external force. Beyond this maximal off-center position $x_{e q}$ becomes closer to the center the higher $u_{0}$ is because the increasing tank-treading motion and $x_{e q}$ is the closer to the wall the larger the external force is. With a weak force and a strong flow e.g. $F_{g}=-10^{-3}$ and $u_{0}>0.7$ the stable position is again the center of the channel despite they are antiparallel.

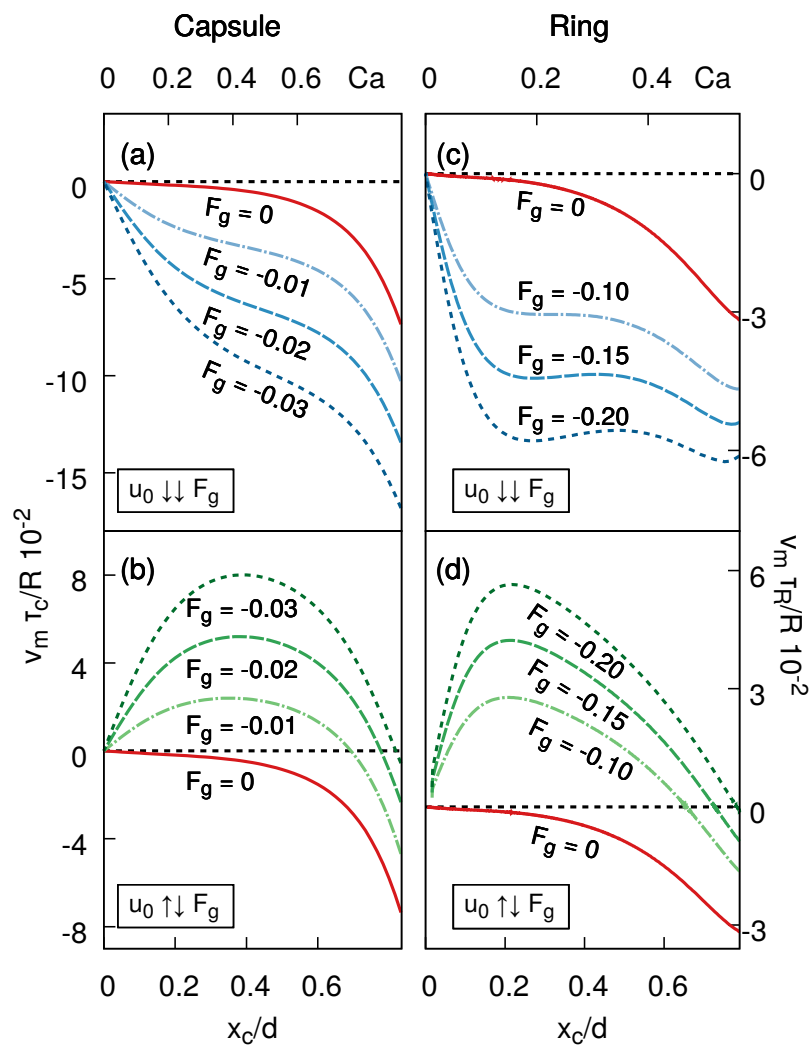

Fig. 5: Migration velocity of soft particles analogous to Fig. 2 . with additional wall interaction. The migration towards the center $\left(v_{m}<0\right)$, observed in the parallel case $\left(u_{0} \downarrow \downarrow F\right.$, upper picture) is increased near the wall. For the antiparallel case ( $u_{0} \uparrow \downarrow F$, lower pictures), the repulsive wall interaction leads to stable positions $\left(v_{m}=0\right)$ between the center and the wall. This effects are again stable for different types of particles and can be seen for both capsules and ring polymers.

Comparison of simulation methods and analytical approximation. We used in our simulations with the Oseen and Blake tensor the assumption that the external force acts on the surface of the capsule. In our analytical approximation we approximate the shape of the capsule as an rotational ellipsoid to calculate the drag. To justify these approximations we compare the results with an Lattice Boltzmann method (LBM) with the BGK collision operator and a singe relaxation time. Flow and particle are coupled by the immersed boundary method. With lattice Boltzmann, it is possible to simulate an external force that acts on the interior of the capsule or on the surface.
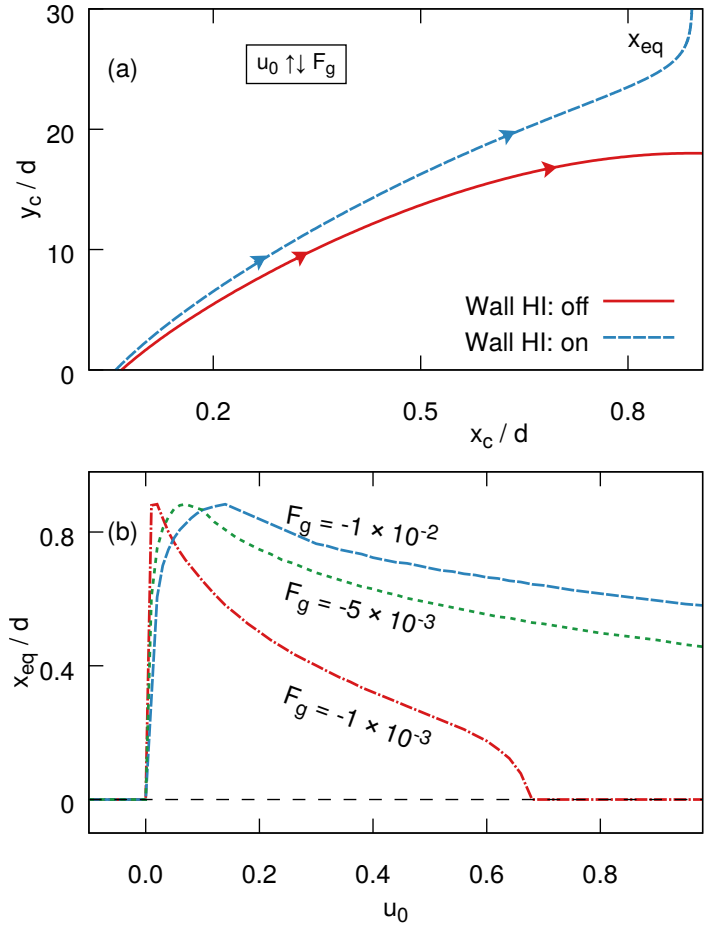

Fig. 6: (Color online) (a) Trajectory of a deformable capsule immersed in a plane Poiseuille flow $\boldsymbol{u}_{0}(\boldsymbol{r})$ between two parallel walls (see also Fig. 1). The flow is antiparallel to the negative buoyancy force $\boldsymbol{F}_{g}$. Without the wall interaction (red) the capsule migrates towards the walls due to $\boldsymbol{F}_{g}$ until it collides with the wall. With wall interaction (green) the migration stops close to the walls at $\frac{x_{c}}{d} \approx 0.85$ because the wall is repulsive (see Fig. 4). This means the capsule reaches a stable equilibrium distance to the wall $x_{e q}$. (b) Equilibrium position $x_{e q}$ for a capsule as function of the flow velocity for three different values of the negative buoyancy forces. Negative values of $u_{0}$ correspond to a flow parallel to the force thus the center of the flow is the stable position. Positive values of $u_{0}$ mean flow and negative buoyancy force are antiparallel which allows stable off center positions. This leads to a transition of $x_{e q}$ from zero to non-zero values at $u_{0}=0$. Beyond a maximum at approximately $u_{0} \approx 0.05$ the stable position $x_{e q}$ is the closer to the center the faster the flow is because of the tank-treading motion. The tank-treading motion which leads to a center migration without $\boldsymbol{F}_{g}$ becomes stronger with higher $u_{0}$. At the values $F_{g}=-10^{-3}$ and $u_{0}>0.5$ the tank-treading dominates and the capsule migrates to the center, as in the case without $\boldsymbol{F}_{g}$. Furthermore a higher force $\boldsymbol{F}_{g}$ means a stable position closer to wall

With the LBM we can compare the analytical approximation, the Oseen and Blake tensor simulation, a LBM simulation with an external force acting on the surface and a second LBM simulation with an external force acting on the interior of the capsule. We determine the migration velocity of a capsule with an external force in an antiparallel directed, bounded Poiseuille flow as function of the lateral position, see Fig. ??. All simulations show that the capsule migrates towards the walls and stops at a certain distance to the wall due to the wall repulsion. Furthermore 
they all display zero migration in the center, a maximum of the migration in the middle between center and wall and a stable, stationary position at approximately $x_{c} \approx 0.85 \mathrm{~d}$. So the simulations agree qualitatively. Quantitatively the simulations differ at the maximum of the migration velocity (approximately a factor of two) but agree well at low capillary numbers close to the center of the channel. This means the Oseen and Blake tensor describes the capsule qualitatively correct.

The analytical approximation agrees well at low capillary numbers at the center of the channel. This is due to the fact that small deformations are assumed. Also the analytical approximation can not reproduce the stationary position because the wall repulsion is not included here. This means the analytical approximations is justified as long as the assumptions of a small capillary number and a position far away from the wall are given.



Fig. 7: The migration velocity $v_{m}$ of a capsule as function of the lateral position $x_{c}$. We compare four different methods: Simulations with the Oseen and Blake-Tensor (purple) where $\boldsymbol{F}_{g}$ acts on the beads (surface of capsule), Lattice-Boltzmann simulations with $\boldsymbol{F}_{g}$ acts on the surface of capsule (red) or on the interior volume (green) and an analytical approximation (blue, see eq. 20). All the simulations show the same qualitative behavior. The approximation of the Oseen-Tensor and the force $\boldsymbol{F}_{g}$ acting on the beads does not change the qualitative behavior of the capsule, just the quantitative values. Also the calculation fits the simulations as long as the assumption of small $\mathrm{Ca}$ and a position far away from the wall (negligible wall interaction) is valid. We used the parameters $d=60$, $u 0=0.01, \tau=1.0$, Density of the Fluid $\rho=1.0, \eta=1.0 / 6.0$, Channel size in flow direction $N_{x}=100$, Channel size in zdirection $N_{z}=100, \kappa=0.001, G=0.001, k_{v}=0.01, b=1$, $\boldsymbol{F}_{g}=-10^{-4} \hat{e}_{x}, a=0.2$.

Summary and conclusions. - The migration of the sinking (elevating) soft particles away from the walls has a similar origin as the lift force observed in linear shear flow 21,23 .

Migration during sedimentation

This cross-streamline migration is more efficient than bulk migration.
By this method we can separate particles with respect to their density and stiffness.

Shear flow

$$
* * *
$$

\section{REFERENCES}

[1] Squires T. M. and Quake S. R., Rev. Mod. Phys., 77 (2005) 978.

[2] KIRBy B. J., Micro- and Nanoscale Fluid Mechanics (Cambridge Univ. Press, Cambridge) 2010.

[3] Nguyen N.-T. and Wereley S. T., Fundamentals and Applications of Microfluidics (Artech House, Boston) 2010.

[4] Whitesides G. M., Nature, 442 (2006) 368.

[5] Popel A. S. and Johnson P. C., Annu. Rev. Fluid Mech., 37 (2005) 43.

[6] Graham M. D., Ann. Rev. Fluid Mech., 43 (2011) 273.

[7] Sackmann E. K., Fulton A. L. and Beebe D. L., $\mathrm{Na}$ ture, 507 (2014) 181.

[8] Dahl J. B., Lin J.-M. G., Muller S. J. and Kumar S., Ann. Rev. Chem. Biomol. Eng., 6 (2015) 293.

[9] Amini H., Lee W. and Carlo D. D., Lap Chip, 14 (2014) 2739.

[10] Geislinger T. M. and Franke T., Adv. Coll. Int. Sci., 208 (2014) 14.

[11] Sajeesh P. and Sen A. K., Microfluid Nanofluid, 17 (2014) 1.

[12] Misbah C., J. Fluid Mech., 760 (2014) 1.

[13] Farutin A., Piasecki T., Slowicka A. M., Misbah C., Wajnryb E. and EkIEL-JeźEwska M. L., Soft Matter, 12 (2016) 7307.

[14] Segré G. and Silberberg A., Nature, 189 (1961) 209.

[15] Shafer R. H., Biophy. Chem., 2 (1974) 185.

[16] Nitsche L. C., AIChE J., 42 (1996) 613.

[17] Ghigliotti G., Rahimian A., Biros G. and Misbah C., Phys. Rev. Lett., 106 (2011) 028101.

[18] Sekhon G., Armstrong R. and Jhon M. S., J. Polymer Sci.:Polymer Phys., 20 (1982) 947.

[19] Brunn P. O., Int. J. Multiphase Flow, 187 (1983) 202.

[20] Jhon M. S. and Freed K. F., J. Polymer Sci.: Polymer Phys., 23 (1985) 255.

[21] Cantat I. and Misbah C., Phys. Rev. Lett., 83 (1999) 880.

[22] Seifert U., Phys. Rev. Lett., 83 (1999) 876.

[23] Abkarian M., Lartigue C. and Viallat A., Phys. Rev. Lett., 88 (2002) 068102.

[24] Ma H. and Graham M. D., Phys. Fluids, 17 (2005) 083103.

[25] Leal L. G., Ann. Rev. Fluid Mech., 12 (1980) 435.

[26] Mandal S., Bandopadhyay A. and Chakraborty S., Phys. Rev. E, 92 (2015) 023002.

[27] Kaoui B., Ristow G. H., Cantat I., Misbah C. and Zimmermann W., Phys. Rev. E, 77 (2008) 021903.

[28] Coupier G., Kaoui B., Podgorski T. and Misbah C., Phys. Fluids, 20 (2008) 111702. 
[29] Doddi S. K. and Bagchi P., Int. J. Multiphas. Flow, 34 (2008) 966.

[30] Farutin A. and Misbah C., Phys. Rev. E, 89 (2011) 042709 .

[31] Watari N. and Larson R. G., Phys. Rev. Lett., 102 (2009) 246001.

[32] Laumann M., Bauknecht P., Gekle S., Kienle D. and Zimmermann W., EPL, 117 (2017) 44001.

[33] Jo I., Huang Y., Zimmermann W. and Kanso E., Phys. Rev. E, 94 (2016) 063116.

[34] Jeffrey R. C. and Pearson J. R. A., J. Fluid Mech., 22 (1965) 721.

[35] Kim Y. W. and Yoo J. Y., Lap Chip, 9 (2009) 1043.

[36] Prohm C. and Stark H., Lap Chip, 14 (2015) 2115.

[37] Zheng J. and Yeung E. S., Anal. Chem., 74 (2002) 4536.

[38] Usta O. B., Butler J. E. and Ladd A. J. C., Phys. Rev. Lett., 98 (2007) 098301.

[39] Feng J., Hu H. H. and Joseph D. D., J. Fluid Mech., 261 (1994) 95.

[40] Blake J. R., Proc. Camb. Philos. Soc., 70 (1971) 303.

[41] Dhont J. K. G., An Introduction to dynamics of colloids (Elsevier, Amsterdam) 1996.

[42] Jones R. B., J. Chem. Phys., 121 (2004) 483.

[43] Barthès-Biesel D., J. Fluid Mech., 113 (1981) 251.

[44] Barthès-Biesel D., Annu. Rev. Fluid Mech., 48 (2016) 25.

[45] Krüger T., Kusumaatmaja H., Kuzmin A., Shardt O., Silva G. and Viggen E. M., The Lattice Boltzmann Method - Principles and Practice (Springer, Berlin) 2016.

[46] Happel J. and Brenner H., Low Reynolds Number Hydrodynamics (Prentice-Hall, Englewood Cliffs) 1981.

[47] Krüger T., Kaoui B. and Harting J., J. Fluid Mech., 751 (2014) 725. 